\title{
Recursive Set-Membership State Estimation over A FlexRay Network
}

\author{
Shuai Liu, Zidong Wang, Licheng Wang, and Guoliang Wei
}

\begin{abstract}
In this paper, we investigate the set-membership state estimation problem for a class of time-varying systems with non-Gaussian noises over a FlexRay network. To mitigate the communication load and improve the flexibility of the data scheduling, the FlexRay protocol governed by both the timeand the event-triggered rules is exploited to regulate the signal transmission in a cyclic fashion. A new expression of the input signal to the state estimator is formulated with intention to account for the effect of the FlexRay protocol. Accordingly, a multi-rate model (orchestrating the sampling/updating rates of the target plant, the sensors and the state estimator) is proposed and then transformed into a single-rate one with the help of the lifting technique and the vector augmentation method. Subsequently, sufficient conditions are provided for the true states to always reside in an ellipsoid at each time instant in the presence of non-Gaussian noises, and such an ellipsoid is then minimized in the matrix-trace sense. An online optimization algorithm is developed to parameterize the estimator gains by means of the solution to certain recursive matrix inequalities. Numerical results demonstrate the validity of the proposed protocol-based set-membership state estimator design scheme.
\end{abstract}

Index Terms-Set-membership state estimation; FlexRay network; Time-varying systems; Multi-rate mechanism; NonGaussian noises.

\section{INTRODUCTION}

Along with the recurring upgrade of the network technology, the so-called networked control systems (NCSs) have been gaining an ever-growing popularity in industry for their distinctive capabilities of remote operation, low cost, and simple installation and maintenance. These prominent features have merited the broad applications of NCSs in various areas including environment sensing, intelligent traffic and smart home, see [7], [9], [13], [14], [18], [27], [42]. Within the academic communities, a great deal of research attention has been devoted towards the security, the reliability, the real-time implementation and the resource utilization of the NCS [23], [24]. In particular, with the increase of the network scale, the

This work was supported in part by the National Natural Science Foundation of China under Grants 61873148, 61873169, 61903253 and 61933007, the Royal Society of the UK, and the Alexander von Humboldt Foundation of Germany. (Corresponding Author: Guoliang Wei.)

S. Liu and G. Wei are with the College of Science, Shanghai Key Lab of Modern Optical System, University of Shanghai for Science and Technology, Shanghai 200093, China. (Emails: liushuai871030@163.com; guoliang. weidusst.edu.cn)

Z. Wang is with the Department of Computer Science, Brunel University London, Uxbridge, Middlesex, UB8 3PH, United Kingdom. (Email: Zidong.Wang@brunel.ac.uk)

L. Wang is with the Shanghai Key Lab of Modern Optical System, the Department of Control Science and Engineering, University of Shanghai for Science and Technology, Shanghai 200093, China. (Email: wanglicheng1217@163. com) inherent restraint on the network resources (e.g. bandwidth and bit rate) have posed great challenges to traditional control or state estimation strategies.

The NCS is now known to be confronted with limited communication capacities that would give rise to unavoidable network-induced phenomena such as data collisions and communication delays [5], [10], [35]. In this context, many efficient schemes have been proposed to either mitigate the network traffic (at the cost of sacrificing certain system performance) or make full use of the incomplete data (to achieve the locally optimal performance indices) [20], [38], [41]. A well-recognized scheme that has been welcomed in industry is the so-called communication protocol whose aim is to regulate the data exchanges over shared channels so as to improve the utilization efficiency of the scarce network resource. So far, a variety of protocol-based control/estimation algorithms have been developed where the communication protocol has been skillfully embedded into the networked systems, see e.g. [4], [15], [26], [36], [37], [47]. Several frequently employed protocols include the stochastic communication protocol (SCP), the Try-Once-Discard protocol (TODP), the Round-Robin protocol (RRP) and the FlexRay protocol (FRP). Further research topics include the extension of our results to 1) other FRP-based state estimation problems, such as the $H_{\infty}$ filtering and the Kalman filtering; and 2) more general systems, such as complex networks, sensor networks and multi-agent systems.

Among the communication protocols, the RRP and the SCP have time-triggered strategies for selecting sensor nodes to access the shared channel, while the selection strategy for the TODP is event-triggered. The FRP, on the other hand, is a hybrid communication protocol that supports both the timeand the event-triggered communications. To be more specific, the FRP provides a time-triggered transmission for the timecritical periodic data in a static segment and a priority-based scheduling rule for the event-triggered signals in a dynamic segment, thereby greatly improving the flexibility and the reliability of the network transmission. Moreover, the FRP works with communication cycles that alternate between a static segment and a dynamic segment. As such, in a FlexRay network, there are two kinds of switching rules executing: 1) the switching among individual static segment (or dynamic segment) and 2) the switching from static (dynamic) segment to dynamic (static) segment. As a result, a fundamental yet open research topic is how to properly characterize these two kinds of switching rules in a unified form especially in the discrete-time setting.

Since its first application to the BMW X5 series in 2006, 
This article has been accepted for publication in a future issue of this journal, but has not been fully edited. Content may change prior to final publication. Citation information: DOI10.1109/TSMC.2021.3071390, IEEE Transactions on Systems, Man, and Cybernetics: Systems

the FRP has attracted particular research attention in a large group of car manufacturers and automotive electronic suppliers [31]. Note that, despite its obvious engineering potentials, only some initial research effort has been made towards the theoretical investigation on the FRP-based control/estimation issues. To name a few, in [43], the stabilization problem has been discussed for a class of continuous-time networked systems over the FlexRay network, and the main results in [43] have been further extended in [44] to the state estimation problem and in [39] for the tracking control issue. As exemplified above, most FRP-related results have been tailored to the continuous-time systems, and the corresponding results on the discrete-time counterparts have been very few despite the prevalent trend of today's digital communication. Consequently, it is of practical significance to look into the control/estimation issues for discrete-time systems under the FRP scheduling, which constitutes one of the motivations of our current research.

In the last few decades, the state estimation or filtering problems have been paid an enduring research interest from the signal processing and control areas. From a mathematical point of view, most of the filtering schemes (e.g. Kalman and $H_{\infty}$ filtering strategies) are customarily referred to as the so-called point estimation schemes that involve the use of measurement data to calculate a single estimation value of the true state, see e.g. [1], [3], [6], [28], [30], [33], [40], [49]. Nevertheless, in many practical problems (e.g. target tracking as well as system guidance and navigation), the target plant is required to fall within a safe region with $100 \%$ confidence, and this has led to the proposal of the so-called set-membership state estimation (SMSE) strategy. In contrast to the point estimation scheme that just obtains an exact estimate value, the SMSE strategy is able to generate an ellipsoid region containing all true states. Till now, a great many SMSE-relevant research results have been found in the literature, see e.g. [19], [46], [48] for some representative works. Nonetheless, when it comes to the FRPbased ellipsoidal estimation, the corresponding results have been very limited (if not none) despite the potential application prospects of both FRP and SMSE in NCSs.

Inspired by the aforementioned discussions, we endeavor to deal with the SMSE problem for a class of time-varying systems under the FRP. In this sense, three inevitable difficulties emerge as follows: 1) how to define the transmission rules for both the static scheduling and the dynamic scheduling; 2) how to provide mathematically precise description of the discrete-time version of the FRP that not only embodies the two kinds of switching rules but also facilitates the subsequent SMSE analysis/synthesis; and 3) how to quantify the impact from the FRP on the estimation performance. To get over these difficulties, we make the following contributions in this paper as summarized below.

- By combining the merits of both the RRP and the TODP, a new mathematical description is proposed to characterize the discrete-time version of the FRP.

- The impact of the FRP on the design of the state estimator is examined in the light of the vector augmentation approach and the multi-rate mechanism.

- A recursive optimization algorithm, which can be imple- mented online, is proposed to acquire a set of optimized ellipsoids containing all possible true states.

The subsequent part of this paper is arranged as follows. Section II formulates the model of the target plant and gives a detailed description of the FRP. By virtue of the lifting technique and the vector augmentation method, a new FRPbased measurement model is described in Section III and the corresponding state estimator is designed in Section IV. In Section $\mathrm{V}$, an optimization problem with certain inequality constraints is put forward for the sake of obtaining an optimized ellipsoid containing all true states at each time instant. Section VI provides a simulation example to verify the effectiveness of the proposed state estimator design scheme. Section VII outlines the conclusions of this paper.

Notations. Throughout this paper, $\mathbb{R}^{n}, \mathbb{R}^{m \times n}$ and $\mathbb{N}^{+}$ denote, respectively, the $n$-dimensional Euclidean space, the set of all $m \times n$ real-valued matrices and the set of al1 non-negative integers. For a matrix $M, M^{T}, M^{-1}$ and $\operatorname{tr}\{M\}$ represent its transpose, inverse and trace, respectively. $I$ stands for the identity matrix of compatible dimension. For symmetric matrices $X$ and $Y$, the notation $X \geq Y$ $(X>Y)$ means that $X-Y$ is non-negative definite (positivedefinite). $\operatorname{diag}\left\{A_{1}, A_{2}, \ldots, A_{n}\right\}$ represents a block diagonal matrix whose diagonal elements are matrices $A_{1}, A_{2}, \ldots, A_{n}$. $\|z\|$ describes the Euclidean norm of a vector $z . \delta(\cdot)$ denotes the delta function, i.e., $\delta(a)=1$ when $a=0$; otherwise $\delta(a)=0 . " \bmod (a, b) "$ denotes the remainder on division of the integer $a$ by the positive integer $b$.

\section{PRoblem Formulation}

\section{A. System model}

The target plant is modeled by the following system:

$$
\left\{\begin{aligned}
x\left(T_{k+1}\right) & =A\left(T_{k}\right) x\left(T_{k}\right)+B\left(T_{k}\right) w\left(T_{k}\right) \\
y\left(T_{k}\right) & =C\left(T_{k}\right) x\left(T_{k}\right)+D\left(T_{k}\right) w\left(T_{k}\right)
\end{aligned}\right.
$$

where $x\left(T_{k}\right) \in \mathbb{R}^{n}$ and $y\left(T_{k}\right) \in \mathbb{R}^{m}$ are, respectively, the system state to be estimated and the measurement output measured by $m$ sensors. $A\left(T_{k}\right), B\left(T_{k}\right), C\left(T_{k}\right)$ and $D\left(T_{k}\right)$ are time-varying matrices with appropriate dimensions. Also, we denote $C\left(T_{k}\right) \triangleq\left[C_{1}^{T}\left(T_{k}\right), C_{2}^{T}\left(T_{k}\right), \ldots, C_{m}^{T}\left(T_{k}\right)\right]^{T}$ and $D\left(T_{k}\right) \triangleq\left[D_{1}^{T}\left(T_{k}\right), D_{2}^{T}\left(T_{k}\right), \ldots, D_{m}^{T}\left(T_{k}\right)\right]^{T}$ with $C_{i}\left(T_{k}\right) \in$ $\mathbb{R}^{1 \times n}$ and $D_{i}\left(T_{k}\right) \in \mathbb{R}^{1 \times w}(i=1,2, \ldots, m)$. Let $h \triangleq$ $T_{k+1}-T_{k}\left(k \in \mathbb{N}^{+}\right)$with $T_{0}=0$ where $h$ is a positive scalar representing the evolution period of the target plant. $w\left(T_{k}\right) \in \mathbb{R}^{w}$ is the exogenous noise belonging to the following ellipsoidal set:

$$
\mathscr{W}\left(T_{k}\right) \triangleq\left\{w\left(T_{k}\right): w^{T}\left(T_{k}\right) W^{-1}\left(T_{k}\right) w\left(T_{k}\right) \leq 1\right\}
$$

with $W\left(T_{k}\right)$ being a given positive-definite matrix sequence.

In practical applications, the communication capacity of a network is usually limited. In this case, simultaneous transmission of large amounts of data would inevitably lead to data collisions. In this paper, with hope to improve both the reliability and the flexibility of data broadcast, the FRP is introduced to schedule the transmission of the $m$ measurement signals from the sensors to the state estimator where only one sensor node is assigned to access the network resource at each time instant. 
This article has been accepted for publication in a future issue of this journal, but has not been fully edited. Content may change prior to final publication. Citation information: DOI10.1109/TSMC.2021.3071390, IEEE Transactions on Systems, Man, and Cybernetics: Systems

\section{B. Discrete-time version of the FlexRay protocol}

Generally speaking, the FlexRay network (i.e., the network scheduled by the FlexRay communication protocol) consists of a series of pre-set communication cycles. As shown in Fig. 1, each communication cycle includes four parts: a static segment, a dynamic segment, a symbol window and a network idle time. The time lengths of these four parts are, respectively, denoted by $R_{1}, R_{2}, R_{3}$ and $R_{4}$. Compared with the static segment and the dynamic segment, the time lengths of the symbol window and the network idle time are very short and usually negligible, i.e. $R_{3}=R_{4}=0$. Also, without loss of generality, we assume that $R_{1}=l h(l \in\{1, \ldots, m\})$ and $R_{2}=h$. Besides, the transmission time of the data packet, which mainly depends on the transmission distance and the size of the packet, is also neglected. To this end, the time length of a communication cycle is $R_{1}+R_{2}+R_{3}+R_{4}=$ $(l+1) h$. In the following, under the discrete-time setting, we shall provide a detailed description on how to elaborately allocate the time of $R_{1}$ and $R_{2}$.

Remark 1: As we know, the transmission time of a data packet is mainly dependent on two factors: the transmission distance and the size of the packet (i.e. the bits occupied by the packet) [43]. Normally, the farther the transmission distance (or the larger the packet size), the longer the transmission time. However, it is worth emphasizing that the FRP is usually customized for the data communication among vehicles' interior devices, where the transmission distance is quite short and thus the resulted impact on the transmission time can be ignored. On the other hand, under the FRP, the maximum data rate of each channel can be up to $10 \mathrm{M} \mathrm{Bit/s}$, which is actually a high-rate communication bus and almost no time delay during the data transmission in a vehicle, that is, the transmission time of the packet can also be neglected compared with an entire communication period. Based on the above discussion, we exclude the influence of the transmission time in this paper.

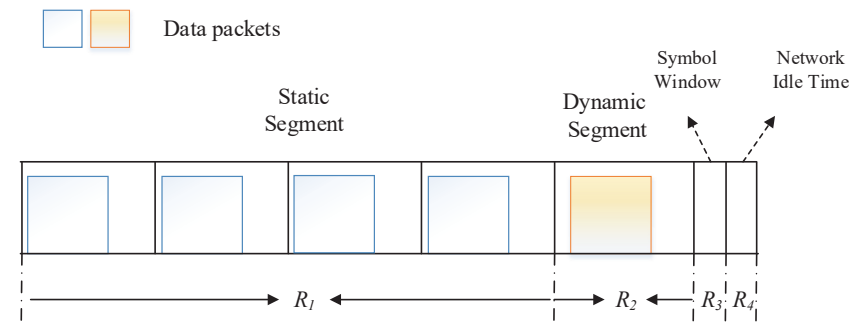

Fig. 1: A communication cycle of the FlexRay network.

For expression convenience, we denote

$$
\begin{aligned}
y\left(T_{k}\right) & \triangleq\left[\left(y^{(1)}\left(T_{k}\right)\right)^{T},\left(y^{(2)}\left(T_{k}\right)\right)^{T}\right]^{T} \\
\bar{y}\left(T_{k}\right) & \triangleq\left[\left(\bar{y}^{(1)}\left(T_{k}\right)\right)^{T},\left(\bar{y}^{(2)}\left(T_{k}\right)\right)^{T}\right]^{T} \\
y^{(1)}\left(T_{k}\right) & \triangleq\left[y_{1}\left(T_{k}\right), \ldots, y_{l}\left(T_{k}\right)\right]^{T} \\
y^{(2)}\left(T_{k}\right) & \triangleq\left[y_{l+1}\left(T_{k}\right), \ldots, y_{m}\left(T_{k}\right)\right]^{T} \\
\bar{y}^{(1)}\left(T_{k}\right) & \triangleq\left[\bar{y}_{1}\left(T_{k}\right), \ldots, \bar{y}_{l}\left(T_{k}\right)\right]^{T} \\
\bar{y}^{(2)}\left(T_{k}\right) & \triangleq\left[\bar{y}_{l+1}\left(T_{k}\right), \ldots, \bar{y}_{m}\left(T_{k}\right)\right]^{T} .
\end{aligned}
$$

It should be pointed out that, in this paper, the RRP and the TODP are, respectively, applied to the static segment and the dynamic segment. More specifically, as shown in Fig. 1, in a communication cycle, the RRP is first carried out in the time length $R_{1}$, and the TODP is then executed in the time length $R_{2}$. Moreover, considering different real-time requirements for different components of measurement signals, those components with the high real-time requirement are scheduled based on the RRP, and other components are scheduled according to the TODP. Without loss of generality, we assume that the first $l$ components $y^{(1)}\left(T_{k}\right) \in \mathbb{R}^{l}$ of $y\left(T_{k}\right)$ are scheduled by the RRP and the remaining $m-l$ components $y^{(2)}\left(T_{k}\right) \in \mathbb{R}^{m-l}$ of $y\left(T_{k}\right)$ are orchestrated by the TODP. Accordingly, let $\bar{y}\left(T_{k}\right)$ be the measurement outputs after network transmission where $\bar{y}^{(1)}\left(T_{k}\right)$ and $\bar{y}^{(2)}\left(T_{k}\right)$ denote the measurement value after the network transmission scheduled by the RRP and the TODP, respectively. More concretely, denote by $\lambda_{T_{k}}$ (or $\sigma_{T_{k}}$ ) the sensor node accessing the transmission right at time $T_{k}$ according to the RRP (or the TODP), which can be determined according to the following rules, separately.

1) Round-Robin protocol is a time-based static scheduling algorithm where the transmission order is governed by the following transmission rule:

$$
\lambda_{T_{k}} \triangleq \bmod \left(\frac{T_{k}}{h}, l\right)+1
$$

with $\lambda_{T_{k}} \in \mathcal{L}_{1} \triangleq\{1, \ldots, l\}$. It implies from (4) that, at time $T_{k}$, only the component $y_{\lambda_{T_{k}}}\left(T_{k}\right)$ of $y\left(T_{k}\right)$ is updated and other components of $y\left(T_{k}\right)$ are set as 0 by means of the zero-input strategy.

2) Try-Once-Discard protocol is an event-based dynamic scheduling algorithm that assigns priorities according to the following specific event:

$$
\sigma_{T_{k}} \triangleq \max _{i=l+1, \ldots, m}\left\{\left(\tilde{y}_{i}^{(2)}\left(T_{k}\right)\right)^{T} Q_{i}\left(T_{k}\right) \tilde{y}_{i}^{(2)}\left(T_{k}\right)\right\}
$$

where $\sigma_{T_{k}} \in \mathcal{L}_{2} \triangleq\{l+1, \ldots, m\}$ and $Q_{i}\left(T_{k}\right)(i \in$ $\left.\mathcal{L}_{2}\right)$ are given positive-definite matrices. Let $\tilde{y}_{i}^{(2)}\left(T_{k}\right) \triangleq$ $y_{i}^{(2)}\left(T_{k}\right)-\bar{y}_{i}^{(2)}\left(T_{k-1}\right)$ and, in terms of the zero-order holders (ZOHs), $\bar{y}_{i}\left(T_{k}\right)\left(i \in \mathcal{L}_{2}\right)$ can be represented as the following form:

$$
\bar{y}_{i}\left(T_{k}\right)=\left\{\begin{array}{l}
y_{i}\left(T_{k}\right), \quad \text { if } i=\sigma_{T_{k}} \\
\bar{y}_{i}\left(T_{k-1}\right) \text { otherwise. }
\end{array}\right.
$$

Having elaborated on the principle of the FRP, we shall provide a detailed mathematical model of the discrete-time version of the FlexRay communication protocol, and then design the FRP-based state estimator.

\section{A NEW MEASUREMENT MODEL}

Because of the cyclic characteristic of the FRP, we are interested in exploring the impact of the FRP on the estimator design in one communication period. In this sense, the difficulties caused by two kinds of switching rules of the FRP can be obviated by setting the update period of $\bar{y}\left(T_{k}\right)$ (i.e. the measurement outputs after network transmission) as the length of one communication cycle (i.e. $(l+1) h)$. Accordingly, for the purpose of facilitating the design of the state estimator, the update period of the state estimator is also set as $(l+1) h$. 
This article has been accepted for publication in a future issue of this journal, but has not been fully edited. Content may change prior to final publication. Citation information: DOI10.1109/TSMC.2021.3071390, IEEE Transactions on Systems, Man, and Cybernetics: Systems

In addition, it is worth noting that the node selected by the TODP might be different, so there should be $m-l$ different communication cycles.

In what follows, the lifting technique [32] is employed to unify the update period of the target plant to be $(l+1) h$.

Lemma 1: The target plant (1) can be transformed into the following form:

$$
\bar{x}\left(t_{k+1}\right)=\bar{A}\left(t_{k}\right) \bar{x}\left(t_{k}\right)+\bar{B}\left(t_{k}\right) \bar{w}\left(t_{k}\right)
$$

where $t_{k+1}-t_{k} \triangleq(l+1) h=g h, t_{0}=0$ and

$$
\begin{aligned}
& \bar{x}\left(t_{k}\right) \triangleq \operatorname{col}\left\{x\left(t_{k-1}+h\right), x\left(t_{k-1}+2 h\right), \ldots, x\left(t_{k}\right)\right\} \\
& \bar{w}\left(t_{k}\right) \triangleq \operatorname{col}\left\{w\left(t_{k}\right), w\left(t_{k}+h\right), \ldots, w\left(t_{k}+(g-1) h\right)\right\} \\
& \bar{A}\left(t_{k}\right) \triangleq \operatorname{col}\left\{\vec{A}_{1}\left(t_{k}\right), \vec{A}_{2}\left(t_{k}\right), \ldots, \vec{A}_{g}\left(t_{k}\right)\right\} \\
& \bar{B}\left(t_{k}\right) \triangleq \operatorname{col}\left\{B_{1}^{(g)}\left(t_{k}\right), B_{2}^{(g-1)}\left(t_{k}\right), \ldots, B_{g}^{(1)}\left(t_{k}\right)\right\} \\
& \vec{A}_{i}\left(t_{k}\right) \triangleq \operatorname{col}^{T}\{\underbrace{0, \ldots, 0}_{g-1}, A_{i}^{T}\left(t_{k}\right)\}, i=1, \ldots, g \\
& A_{g-b+1}\left(t_{k}\right) \triangleq \prod_{i=b}^{g} A\left(t_{k+1}-i h\right), b=1, \ldots, g \\
& B_{b}^{(r)}\left(t_{k}\right) \triangleq \\
& \left\{\begin{array}{l}
{[B\left(t_{k}\right) \underbrace{0, \ldots, 0}_{g-1}, b=1, r=g} \\
{[\vec{B}_{1}^{(r)}\left(t_{k}\right) \ldots \vec{B}_{b-1}^{(r)}\left(t_{k}\right) B\left(t_{k}+(b-1) h\right) \underbrace{0, \ldots, 0}_{g-b}]} \\
b=2, \ldots, g, r=g-b+1
\end{array}\right. \\
& \vec{B}_{j}^{(r)}\left(t_{k}\right) \triangleq \prod_{i=r}^{g-j} A\left(t_{k+1}-i h\right) B\left(t_{k}+(j-1) h\right) \\
& j=1, \ldots, b-1 .
\end{aligned}
$$

Proof: First, according to (1), one has

$$
x\left(t_{k}+h\right)=A_{1}\left(t_{k}\right) x\left(t_{k}\right)+B_{1}^{(g)}\left(t_{k}\right) \bar{w}\left(t_{k}\right)
$$

and then

$$
\begin{aligned}
& x\left(t_{k}+2 h\right) \\
= & A\left(t_{k}+h\right) x\left(t_{k}+h\right)+\tilde{B}\left(t_{k}+h\right) \bar{w}\left(t_{k}\right) \\
= & A\left(t_{k}+h\right) A_{1}\left(t_{k}\right) x\left(t_{k}\right)+A\left(t_{k}+h\right) B_{1}^{(g)}\left(t_{k}\right) \bar{w}\left(t_{k}\right) \\
& +\tilde{B}\left(t_{k}+h\right) \bar{w}\left(t_{k}\right) \\
= & A_{2}\left(t_{k}\right) x\left(t_{k}\right)+B_{2}^{(g-1)}\left(t_{k}\right) \bar{w}\left(t_{k}\right)
\end{aligned}
$$

where, for $i=1, \ldots, g-1$,

$$
\tilde{B}\left(t_{k}+i h\right) \triangleq[\underbrace{0, \ldots, 0}_{i}, B\left(t_{k}+i h\right), \underbrace{0, \ldots, 0}_{g-1-i}] .
$$

Along the similar line, one further has

$$
x\left(t_{k+1}-h\right)=A_{g-1}\left(t_{k}\right) x\left(t_{k}\right)+B_{g-1}^{(2)}\left(t_{k}\right) \bar{w}\left(t_{k}\right)
$$

and, therefore,

$$
\begin{aligned}
& x\left(t_{k+1}\right) \\
= & A\left(t_{k+1}-h\right) x\left(t_{k+1}-h\right)+\tilde{B}\left(t_{k+1}-h\right) \bar{w}\left(t_{k}\right) \\
= & A\left(t_{k+1}-h\right)\left(A_{g-1}\left(t_{k}\right) x\left(t_{k}\right)+B_{g-1}^{(2)}\left(t_{k}\right) \bar{w}\left(t_{k}\right)\right) \\
& +\tilde{B}\left(t_{k+1}-h\right) \bar{w}\left(t_{k}\right) \\
= & A_{g}\left(t_{k}\right) x\left(t_{k}\right)+B_{g}^{(1)}\left(t_{k}\right) \bar{w}\left(t_{k}\right) .
\end{aligned}
$$

From the definition of $\bar{x}\left(T_{k}\right)$, one obtains the compact form (7), which completes the proof of the lemma.

Remark 2: Three observations can be made so far as follows: 1) the sampling period and the transmission period of the measurement output are still $h ; 2$ ) the update period of the state estimator (to be devised later) is set as $(l+1) h$, which is the same as the time length of one communication cycle of the FlexRay network; and 3) the lifting technique has turned out to be an effective approach to addressing the asynchronous sampling/update issue, see e.g. [11].

Due to the introduction of the FRP, at each communication instant, only one sensor node is able to access the network and, accordingly, only one component of the measurement output $y\left(T_{k}\right)$ is updated. In view of this, the following lemma is introduced to derive an update formula of the measurement output (i.e. $\left.\bar{y}\left(t_{k}\right)\right)$ after the FlexRay network.

Lemma 2: Considering the RRP rule (4) and the TODP rule (5), $\bar{y}\left(t_{k}\right)$ can be expressed as

$$
\begin{aligned}
\bar{y}\left(t_{k}\right)= & \hat{C}\left(t_{k}\right) \bar{x}\left(t_{k}\right)+\breve{D}\left(t_{k}\right) \bar{w}\left(t_{k}\right) \\
& +\mathcal{I}_{2}\left(I-\Phi_{\sigma_{t_{k}+l h}}\right) \bar{y}^{(2)}\left(t_{k-1}\right)
\end{aligned}
$$

where, for $i=l+1, \ldots, m, j=2, \ldots, l$ and $r=1, \ldots, l$,

$$
\begin{aligned}
& \mathcal{I}_{1} \triangleq\left[I_{l \times l}, 0_{(m-l) \times l}^{T}\right]^{T} \\
& \mathcal{I}_{2} \triangleq\left[0_{l \times(m-l)}^{T}, I_{(m-l) \times(m-l)}\right]^{T} \\
& \hat{C}\left(t_{k}\right) \triangleq \mathcal{I}_{1} \tilde{C}\left(t_{k}\right)+\mathcal{I}_{2} \dot{C}\left(t_{k}\right) \\
& \breve{D}\left(t_{k}\right) \triangleq \mathcal{I}_{1} \tilde{D}\left(t_{k}\right)+\mathcal{I}_{2} \dot{D}\left(t_{k}\right) \\
& \tilde{C}\left(t_{k}\right) \triangleq \tilde{C}_{1}\left(t_{k}\right)+\tilde{C}_{2}\left(t_{k}\right) \bar{A}\left(t_{k}\right) \\
& \tilde{D}\left(t_{k}\right) \triangleq \tilde{C}_{2}\left(t_{k}\right) \bar{B}\left(t_{k}\right)+\tilde{D}_{1}\left(t_{k}\right) \\
& \dot{C}\left(t_{k}\right) \triangleq \Phi_{\sigma_{t_{k}+l h}} \check{C}\left(t_{k}+l h\right) \bar{A}\left(t_{k}\right) \\
& \dot{D}\left(t_{k}\right) \triangleq \Phi_{\sigma_{t_{k}+l h}} \check{C}\left(t_{k}+l h\right) \bar{B}\left(t_{k}\right)+\Phi_{\sigma_{t_{k}+l h}} \check{D}\left(t_{k}+l h\right) \\
& \check{C}\left(t_{k}+l h\right) \triangleq\left[\check{C}_{l+1}^{T}\left(t_{k}+l h\right), \ldots, \check{C}_{m}^{T}\left(t_{k}+l h\right)\right]_{(m-l) \times(g n)}^{T} \\
& \check{D}\left(t_{k}+l h\right) \triangleq\left[\check{D}_{l+1}^{T}\left(t_{k}+l h\right), \ldots, \check{D}_{m}^{T}\left(t_{k}+l h\right)\right]_{(m-l) \times g w}^{T} \\
& \Phi_{\sigma_{t_{k}+l h}} \triangleq \operatorname{diag}\left\{\delta\left(l+1-\sigma_{t_{k}+l h}\right), \ldots, \delta\left(m-\sigma_{t_{k}+l h}\right)\right\} \\
& \check{C}_{i}\left(t_{k}+l h\right) \triangleq[\underbrace{0, \ldots, 0}_{g-2}, C_{i}\left(t_{k}+l h\right), 0]_{1 \times(g n)} \\
& \check{D}_{i}\left(t_{k}+l h\right) \triangleq[\underbrace{0, \ldots, 0}_{g-1}, D_{i}\left(t_{k}+l h\right)]_{1 \times(g w)} \\
& \tilde{C}_{1}\left(t_{k}\right) \triangleq[\vec{C}_{1}^{T}\left(t_{k}\right), \underbrace{0, \ldots, 0}_{l-1}]_{l \times(g n)}^{T} \\
& \tilde{C}_{2}\left(t_{k}\right) \triangleq\left[0, \vec{C}_{2}^{T}\left(t_{k}+h\right), \ldots, \vec{C}_{l}^{T}\left(t_{k}+(l-1) h\right)\right]_{l \times(g n)}^{T} \\
& \tilde{D}_{1}\left(t_{k}\right) \triangleq\left[\vec{D}_{1}^{T}\left(t_{k}+h\right), \ldots, \vec{D}_{l}^{T}\left(t_{k}+(l-1) h\right)\right]_{l \times(g w)}^{T}
\end{aligned}
$$


This article has been accepted for publication in a future issue of this journal, but has not been fully edited. Content may change prior to final publication. Citation information: DOI10.1109/TSMC.2021.3071390, IEEE Transactions on Systems, Man, and Cybernetics: Systems

$$
\begin{aligned}
& \vec{C}_{1}\left(t_{k}\right) \triangleq[\underbrace{0, \ldots, 0}_{g-1}, C_{1}\left(t_{k}\right)] \\
& \vec{C}_{j}\left(t_{k}+(j-1) h\right) \triangleq[\underbrace{0, \ldots, 0}_{j-2}, C_{j}\left(t_{k}+(j-1) h\right), \underbrace{0, \ldots, 0}_{g+1-j}]_{1 \times(g n)} \\
& \vec{D}_{r}\left(t_{k}+(r-1) h\right) \\
& \triangleq[\underbrace{0, \ldots, 0}_{r-1}, D_{r}\left(t_{k}+(r-1) h\right), \underbrace{0, \ldots, 0}_{g-r}]_{1 \times(g w)} .
\end{aligned}
$$

Proof: According to the RRP (4), $\bar{y}^{(1)}\left(t_{k+1}\right)$ can be computed as

$$
\bar{y}^{(1)}\left(t_{k}\right)=\tilde{C}\left(t_{k}\right) \bar{x}\left(t_{k}\right)+\tilde{D}\left(t_{k}\right) \bar{w}\left(t_{k}\right) .
$$

Subsequently, based on the TODP (5), one has

$$
\begin{aligned}
\bar{y}^{(2)}\left(t_{k}\right)= & \Phi_{\sigma_{t_{k}+l h}} y^{(2)}\left(t_{k}+l h\right) \\
& +\left(I-\Phi_{\sigma_{t_{k}+l h}}\right) \bar{y}^{(2)}\left(t_{k-1}\right) \\
= & \dot{C}\left(t_{k}\right) \bar{x}\left(t_{k}\right)+\dot{D}\left(t_{k}\right) \bar{w}\left(t_{k}\right) \\
& +\left(I-\Phi_{\sigma_{t_{k}+l h}}\right) \bar{y}^{(2)}\left(t_{k-1}\right) .
\end{aligned}
$$

It is obvious from (13)-(14) that

$$
\bar{y}\left(t_{k}\right)=\mathcal{I}_{1} \bar{y}^{(1)}\left(t_{k}\right)+\mathcal{I}_{2} \bar{y}^{(2)}\left(t_{k}\right),
$$

which implies that (12) holds, thereby ending the proof of this lemma.

Remark 3: It can be observed from (12) that the measurement output (after being transmitted via a FRP-based network) can be reduced to that for 1) the RRP case when $\mathcal{I}_{1}=I_{m \times m}$ and $\mathcal{I}_{2}=0$ and 2$)$ the TODP case when $\mathcal{I}_{1}=0$ and $\mathcal{I}_{2}=I_{m \times m}$. We conclude here that the mathematical description of the FRP proposed in this paper is more general than the individual RRP or TODP, both of which have been widely deployed in industry.

\section{FlexRAy-protocol-BASEd estimator Design}

In this section, in order to deal with the term $\bar{y}^{(2)}\left(t_{k-1}\right)$ caused by the ZOH strategy in (12), the augmented system, which aggregates the system state $\bar{x}\left(t_{k}\right)$ and the holding term $\bar{y}^{(2)}\left(t_{k-1}\right)$, is obtained as follows:

$$
\left\{\begin{array}{l}
\tilde{x}\left(t_{k+1}\right)=\breve{A}\left(t_{k}\right) \tilde{x}\left(t_{k}\right)+\breve{B}\left(t_{k}\right) \bar{w}\left(t_{k}\right) \\
\bar{y}\left(t_{k+1}\right)=\breve{C}\left(t_{k}\right) \tilde{x}\left(t_{k}\right)+\breve{D}\left(t_{k}\right) \bar{w}\left(t_{k}\right)
\end{array}\right.
$$

where

$$
\begin{aligned}
& \tilde{x}\left(t_{k}\right) \triangleq\left[\bar{x}^{T}\left(t_{k}\right),\left(\bar{y}^{(2)}\left(t_{k-1}\right)\right)^{T}\right]^{T} \\
& \breve{A}\left(t_{k}\right) \triangleq\left[\begin{array}{cc}
\bar{A}\left(t_{k}\right) & 0 \\
\dot{C}\left(t_{k}\right) & I-\Phi_{\sigma_{t_{k}+l h}}
\end{array}\right] \\
& \breve{B}\left(t_{k}\right) \triangleq\left[\begin{array}{l}
\bar{B}\left(t_{k}\right) \\
\dot{D}\left(t_{k}\right)
\end{array}\right] \\
& \breve{C}\left(t_{k}\right) \triangleq\left[\begin{array}{ll}
\hat{C}\left(t_{k}\right) & \mathcal{I}_{2}\left(I-\Phi_{\sigma_{t_{k}+l h}}\right)
\end{array}\right] .
\end{aligned}
$$

Next, according to (12) and (16), the following time-varying state estimator is constructed:

$$
\hat{x}\left(t_{k+1}\right)=\breve{A}\left(t_{k}\right) \hat{x}\left(t_{k}\right)+L\left(t_{k}\right)\left(\bar{y}\left(t_{k}\right)-\breve{C}\left(t_{k}\right) \hat{x}\left(t_{k}\right)\right)
$$

where $\hat{x}\left(t_{k}\right) \in \mathbb{R}^{g n+m-l}$ is the estimate of $\tilde{x}\left(t_{k}\right)$ and $L\left(t_{k}\right) \in$ $\mathbb{R}^{(g n+m-l) \times m}$ is the time-varying state estimator parameter to be determined.

For the considered SMSE problem, in this paper, we devote to achieving the following two objectives.

1) Design appropriate time-varying state estimator parameters $L\left(t_{k}\right)$ in (17) such that, for non-Gaussian noises $w\left(t_{k}\right) \in \mathscr{W}\left(t_{k}\right)$ and the FRP (12), all possible true states fall within an ellipsoid, that is, there exists a sequence of positive-definite matrices $P\left(t_{k+1}\right)$ satisfying

$$
\begin{aligned}
\mathscr{E}\left(t_{k+1}\right) \triangleq\{ & \tilde{x}\left(t_{k+1}\right) \in \mathbb{R}^{g n+m-l} \mid \\
& \left(\tilde{x}\left(t_{k+1}\right)-\hat{x}\left(t_{k+1}\right)\right)^{T} P^{-1}\left(t_{k+1}\right) \\
& \left.\times\left(\tilde{x}\left(t_{k+1}\right)-\hat{x}\left(t_{k+1}\right)\right) \leq 1\right\}
\end{aligned}
$$

under the initial condition

$$
\begin{gathered}
\mathscr{E}(0) \triangleq\left\{\tilde{x}(0) \in \mathbb{R}^{g n+m-l} \mid(\tilde{x}(0)-\hat{x}(0))^{T}\right. \\
\left.\times P^{-1}(0)(\tilde{x}(0)-\hat{x}(0)) \leq 1\right\}
\end{gathered}
$$

where $\hat{x}(0)$ and $P(0)$ are, respectively, the given initial state and initial positive-definite matrix. Especially, for the ellipsoid $\mathscr{E}\left(t_{k+1}\right), \hat{x}\left(t_{k+1}\right)$ denotes its center whereas $P\left(t_{k+1}\right)$ determines its radius.

2) At each time instant, minimize $P\left(t_{k+1}\right)$ in the sense of the matrix trace so as to obtain an optimized ellipsoid.

Remark 4: It can be found that the main difficulties caused by the FRP lie in the joint appearance of 1) two kinds of communication mechanisms (i.e. time- and event-triggering mechanisms) and 2) two kinds of time scales (i.e. $t_{k}$ and $T_{k}$ ), which would largely increase the complexity of the state estimator design. To get rid of the difficulty, in the sequel, we propose to use a unified measurement model to enforce these two kinds communication mechanisms, and then adopt the lifting technique to transform the multi-rate model to a single-rate one.

\section{MAin Results}

This section is mainly devoted to the establishment of sufficient conditions ensuring that the augmented system dynamics (7) achieves the objective (18) under the initial condition (19). At the same time, an explicit parameterization of the state estimator gains is obtained in light of the solution to certain recursive matrix inequality. Then, an optimized ellipsoid confining all possible true states is derived in the sense of the matrix trace by solving an optimization problem with certain constraint conditions.

Theorem 1: Consider the augmented system (7), the FlexRay communication protocol (12) and the time-varying state estimator (17). The ellipsoidal constraint (18) is satisfied under the initial condition (19) if there exist positive-definite matrices $P\left(t_{k+1}\right)$, real-valued matrices $L\left(t_{k}\right)$, positive scalars $\varepsilon_{i}\left(t_{k}\right)(i=1,2)$ and $\varrho_{j}\left(t_{k}\right)(j=l+1, \ldots, m)$ such that the following recursive matrix inequality

$$
\left[\begin{array}{cc}
\Omega\left(t_{k}\right)-\Xi\left(t_{k}\right) & * \\
\Gamma\left(t_{k}\right) & -P\left(t_{k+1}\right)
\end{array}\right]<0
$$


holds, where

$$
\begin{aligned}
\Omega\left(t_{k}\right) \triangleq & \operatorname{diag}\left\{-1+\varepsilon_{1}\left(t_{k}\right)+g \varepsilon_{2}\left(t_{k}\right),-\varepsilon_{1}\left(t_{k}\right) I,\right. \\
& \left.-\varepsilon_{2}\left(t_{k}\right) \bar{W}^{-1}\left(t_{k}\right)\right\} \\
\Xi\left(t_{k}\right) \triangleq & \sum_{j=l+1}^{m} \varrho_{j}\left(t_{k}\right) \Sigma^{T}\left(t_{k}\right) \\
& \times\left(\hat{Q}_{j}\left(t_{k}+l h\right)-\hat{Q}_{\sigma_{t_{k}+l h}}\left(t_{k}+l h\right)\right) \Sigma\left(t_{k}\right) \\
\hat{Q}\left(t_{k}\right) \triangleq & \operatorname{diag}\left\{Q_{l+1}\left(t_{k}\right), \ldots, Q_{m}\left(t_{k}\right)\right\} \\
\hat{Q}_{j}\left(t_{k}\right) \triangleq & \hat{Q}\left(t_{k}\right) \Phi_{j}, j=l+1, \ldots, m \\
\Sigma\left(t_{k}\right) \triangleq & {\left[\dot{C}\left(t_{k}\right) \hat{x}\left(t_{k}\right), \dot{C}\left(t_{k}\right) R\left(t_{k}\right), \dot{D}\left(t_{k}\right)\right] } \\
\dot{C}\left(t_{k}\right) \triangleq & {\left[\check{C}\left(t_{k}+l h\right) \bar{A}\left(t_{k}\right),-I\right] } \\
\dot{D}\left(t_{k}\right) \triangleq & \check{C}\left(t_{k}+l h\right) \bar{B}\left(t_{k}\right)+\check{D}\left(t_{k}+l h\right) \\
\bar{W}\left(t_{k}\right) \triangleq & \operatorname{diag}\left\{W\left(t_{k}\right), W\left(t_{k}+h\right), \ldots, W\left(t_{k}+l h\right)\right\} \\
\Gamma\left(t_{k}\right) \triangleq & {\left[0, \quad\left(\breve{A}\left(t_{k}\right)-L\left(t_{k}\right) \breve{C}\left(t_{k}\right)\right) R\left(t_{k}\right),\right.} \\
& \left.\breve{B}\left(t_{k}\right)-L\left(t_{k}\right) \breve{D}\left(t_{k}\right)\right] .
\end{aligned}
$$

Proof: The mathematical induction is applied to prove this theorem.

First, we need to verify that the ellipsoidal constraint is true at the initial instant $t_{0}=0$, and this is evident from (19).

Second, assume that the condition (18) holds at time instant $t_{k}$. It remains to confirm that, at time instant $t_{k+1}$, the condition (18) also holds.

It is easy to see from $\tilde{x}\left(t_{k}\right) \in \mathscr{E}\left(t_{k}\right)$ that there exists a vector $u\left(t_{k}\right)$ satisfying $\left\|u\left(t_{k}\right)\right\| \leq 1$ such that

$$
e\left(t_{k}\right) \triangleq \tilde{x}\left(t_{k}\right)-\hat{x}\left(t_{k}\right)=R\left(t_{k}\right) u\left(t_{k}\right)
$$

where $R\left(t_{k}\right)$ is the factorization of $P\left(t_{k}\right)=R\left(t_{k}\right) R^{T}\left(t_{k}\right)$.

It is derived from (7) and (17) that

$$
\begin{aligned}
e\left(t_{k+1}\right)= & \left(\breve{A}\left(t_{k}\right)-L\left(t_{k}\right) \breve{C}\left(t_{k}\right)\right) R\left(t_{k}\right) u\left(t_{k}\right) \\
& +\left(\breve{B}\left(t_{k}\right)-L\left(t_{k}\right) \breve{D}\left(t_{k}\right)\right) \bar{w}\left(t_{k}\right) .
\end{aligned}
$$

Defining

$$
\theta\left(t_{k}\right) \triangleq\left[\begin{array}{lll}
1 & u^{T}\left(t_{k}\right) & \bar{w}^{T}\left(t_{k}\right)
\end{array}\right]^{T},
$$

one has

$$
e\left(t_{k+1}\right)=\Gamma\left(t_{k}\right) \theta\left(t_{k}\right) .
$$

According to the boundedness condition (2) of nonGaussian noises, it is easy to see that

$$
\bar{w}^{T}\left(t_{k}\right) \bar{W}^{-1}\left(t_{k}\right) \bar{w}\left(t_{k}\right) \leq g .
$$

Based on the constraint conditions $\left\|u\left(t_{k}\right)\right\| \leq 1$ and (25), we have

$$
\left\{\begin{array}{l}
\theta^{T}\left(t_{k}\right) \operatorname{diag}\{-1, I, 0\} \theta\left(t_{k}\right) \leq 0 \\
\theta^{T}\left(t_{k}\right) \operatorname{diag}\left\{-g, 0, \bar{W}^{-1}\left(t_{k}\right)\right\} \theta\left(t_{k}\right) \leq 0 .
\end{array}\right.
$$

In the following, we shall handle the selection condition of the TODP. First, the condition (5) can be rewritten as

$$
\begin{aligned}
& \left(\tilde{y}_{\sigma_{t_{k}}+l h}^{(2)}\left(t_{k}+l h\right)\right)^{T} Q_{\sigma_{t_{k}+l h}}\left(t_{k}+l h\right) \\
& \times \tilde{y}_{\sigma_{t_{k}+l h}}^{(2)}\left(t_{k}+l h\right) \\
\geq & \left(\tilde{y}_{j}^{(2)}\left(t_{k}+l h\right)\right)^{T} Q_{j}\left(t_{k}+l h\right) \tilde{y}_{j}^{(2)}\left(t_{k}+l h\right)
\end{aligned}
$$

for $j \in \mathcal{L}_{2}$, and therefore one derives

$$
\begin{aligned}
& \left(y^{(2)}\left(t_{k}+l h\right)-\bar{y}^{(2)}\left(t_{k}\right)\right)^{T} \hat{Q}_{\sigma_{t_{k}+l h}}\left(t_{k}+l h\right) \\
& \times\left(y^{(2)}\left(t_{k}+l h\right)-\bar{y}^{(2)}\left(t_{k}\right)\right) \\
\geq & \left(y^{(2)}\left(t_{k}+l h\right)-\bar{y}^{(2)}\left(t_{k}\right)\right)^{T} \hat{Q}_{j}\left(t_{k}+l h\right) \\
& \times\left(y^{(2)}\left(t_{k}+l h\right)-\bar{y}^{(2)}\left(t_{k}\right)\right) .
\end{aligned}
$$

Then, it is not difficult to see that

$$
\begin{aligned}
& y^{(2)}\left(t_{k}+l h\right)-\bar{y}^{(2)}\left(t_{k}\right) \\
= & \check{C}\left(t_{k}+l h\right) \bar{A}\left(t_{k}\right) \bar{x}\left(t_{k}\right)+\check{C}\left(t_{k}+l h\right) \bar{B}\left(t_{k}\right) \bar{w}\left(t_{k}\right) \\
& +\check{D}\left(t_{k}+l h\right) \bar{w}\left(t_{k}\right)-\bar{y}^{(2)}\left(t_{k}\right) .
\end{aligned}
$$

By some simple manipulations, one further has

$$
\begin{aligned}
& \left(\check{C}\left(t_{k}+l h\right) \bar{A}\left(t_{k}\right) \bar{x}\left(t_{k}\right)+\dot{D}\left(t_{k}\right) \bar{w}\left(t_{k}\right)-\bar{y}^{(2)}\left(t_{k}\right)\right)^{T} \\
& \times\left(\hat{Q}_{j}\left(t_{k}\right)-\hat{Q}_{\sigma_{t_{k}+l h}}\left(t_{k}+l h\right)\right) \\
& \times\left(\check{C}\left(t_{k}+l h\right) \bar{A}\left(t_{k}\right) \bar{x}\left(t_{k}\right)+\dot{D}\left(t_{k}\right) \bar{w}\left(t_{k}\right)-\bar{y}^{(2)}\left(t_{k}\right)\right) \\
& \leq 0,
\end{aligned}
$$

which implies that

$$
\begin{aligned}
& \theta^{T}\left(t_{k}\right) \Sigma^{T}\left(t_{k}\right)\left(\hat{Q}_{j}\left(t_{k}+l h\right)-\hat{Q}_{\sigma_{t_{k}+l h}}\left(t_{k}+l h\right)\right) \\
& \times \Sigma\left(t_{k}\right) \theta\left(t_{k}\right) \leq 0 .
\end{aligned}
$$

By virtue of the S-procedure [2], the ellipsoidal constraint (18) is true at time instant $t_{k+1}$ if there exist positive scalars $\varepsilon_{i}\left(t_{k}\right)(i=1,2)$ and $\varrho_{j}\left(t_{k}\right)(j=l+1, \ldots, m)$ such that the following inequality constraint is met:

$$
\begin{aligned}
& \theta^{T}\left(t_{k}\right) \Gamma^{T}\left(t_{k}\right) P^{-1}\left(t_{k+1}\right) \Gamma\left(t_{k}\right) \theta\left(t_{k}\right) \\
& -\theta^{T}\left(t_{k}\right) \operatorname{diag}\{1,0,0\} \theta\left(t_{k}\right) \\
& -\varepsilon_{1}\left(t_{k}\right) \theta^{T}\left(t_{k}\right) \operatorname{diag}\{-1, I, 0\} \theta\left(t_{k}\right) \\
& -\varepsilon_{2}\left(t_{k}\right) \theta^{T}\left(t_{k}\right) \operatorname{diag}\left\{-g, 0, \bar{W}^{-1}\left(t_{k}\right)\right\} \theta\left(t_{k}\right) \\
& -\sum_{j=l+1}^{m} \varrho_{j}\left(t_{k}\right) \theta^{T}\left(t_{k}\right) \Sigma^{T}\left(t_{k}\right) \\
& \times\left(\hat{Q}_{j}\left(t_{k}+l h\right)-\hat{Q}_{\sigma_{t_{k}+l h}}\left(t_{k}+l h\right)\right) \Sigma\left(t_{k}\right) \theta\left(t_{k}\right) \\
& \leq 0 .
\end{aligned}
$$

It is easily confirmed that the condition (32) is guaranteed by the following inequality:

$$
\begin{aligned}
& \Gamma^{T}\left(t_{k}\right) P^{-1}\left(t_{k+1}\right) \Gamma\left(t_{k}\right)-\operatorname{diag}\{1,0,0\} \\
& -\varepsilon_{1}\left(t_{k}\right) \operatorname{diag}\{-1, I, 0\} \\
& -\varepsilon_{2}\left(t_{k}\right) \operatorname{diag}\left\{-g, 0, \bar{W}^{-1}\left(t_{k}\right)\right\} \\
& -\sum_{j=l+1}^{m} \varrho_{j}\left(t_{k}\right) \Sigma^{T}\left(t_{k}\right) \\
& \times\left(\hat{Q}_{j}\left(t_{k}+l h\right)-\hat{Q}_{\sigma_{t_{k}+l h}}\left(t_{k}+l h\right)\right) \Sigma\left(t_{k}\right) \\
& \leq 0
\end{aligned}
$$

By means of the Schur Complement Lemma [2], (33) can be ensured by (20), which verifies the constraint condition (18) at time instant $t_{k+1}$. According to the mathematical induction, we conclude that the constraint condition (18) holds for any $k \in \mathbb{N}^{+}$. 
This article has been accepted for publication in a future issue of this journal, but has not been fully edited. Content may change prior to final publication. Citation information: DOI10.1109/TSMC.2021.3071390, IEEE Transactions on Systems, Man, and Cybernetics: Systems

Having obtained a sufficient condition to ensure all possible true states entering into the ellipsoid (18), we are now in a position to propose an optimization problem to minimize such an ellipsoid in the sense of the matrix trace.

Corollary 1: Consider the augmented system (7) with the FlexRay communication protocol (12) and the time-varying state estimator (17). The ellipsoidal set $\mathscr{W}\left(t_{k+1}\right)$ is minimized if there exist positive-definite matrices $P\left(t_{k+1}\right)$, realvalued matrices $L\left(t_{k}\right)$, positive scalars $\varepsilon_{i}\left(t_{k}\right)(i=1,2)$ and $\varrho_{j}\left(t_{k}\right)(j=l+1, \ldots, m)$ such that the following optimization problem

$$
\text { OP : } \quad \min \operatorname{tr}\left\{P\left(t_{k+1}\right)\right\}
$$

is solvable subject to the inequality constraint (20).

Now, in order to show the realizability of the proposed SMSE scheme, the following algorithm (Algorithm 1) is developed.

Algorithm 1 : FlexRay-Protocol-Based Ellipsoidal State Estimator Design Algorithm

Step 1. Initialize the parameters $P(0), x(0)$ and $\hat{x}(0)$ such that the initial constraint condition (19) is satisfied. Set $k=0$ and the maximum step $N$.

Step 2. Based on $P\left(t_{k}\right)=R\left(t_{k}\right) R^{T}\left(t_{k}\right)$, calculate matrix factorizations $R\left(t_{k}\right)$.

Step 3. Solve optimization problem (34) according to the FlexRay scheduling protocol (12). Then, the state estimator parameter $L\left(t_{k}\right)$ and the positive-definite matrix $P\left(t_{k+1}\right)$ can be obtained, respectively. Subsequently, $\hat{x}\left(t_{k+1}\right)$ is computed by (17).

Step 4. Set $k=k+1$. If $k>N$ stop, else go back to Step 2.

Remark 5: Over the past sixty years or so, the SMSE has received considerable research interests, and a great number of achievements have been reported in the literature, see, e.g. [34], and the references therein. Particularly, in the last thirty years, with the rise of networked control systems, the traditional SMSE has been extended to the networked framework and some network-induced phenomena (e.g. packet dropouts, time-delay) have been considered in the SMSE, see, e.g. [21], [45]. It is notable that, to cope with those networkinduced phenomena caused by limited network bandwidths, some transmission protocols have been applied to the SMSE that include the event-triggering protocol, the RRP and the TODP, see [8], [25]. It should be pointed out that, almost all existing works on the protocol-based SMSE problem have exclusively concerned with only the single-protocol-based transmission manner, which is not always competent for a rather complicate communication schedule task in certain practical scenarios. In this paper, to fully absorb the merits of those existing protocols, we combine the existing protocols (RRP and TODP) into a unified framework and make the first attempt to investigate the FRP-based SMSE problem.

Remark 6: Unlike the existing results which are dependent on either the time-triggering pattern or the event-triggering pattern, the employed FRP follows a hybrid communication style under which these two kinds of triggering schemes work alternatively, thus combining the advantages of both schemes. Also, to comply with today's digital communication way, the discrete-time version of the FRP is put forward, and the SMSE strategy is adopted to ensure that all possible true states are confined within an optimized ellipsoid. It should be noted that Algorithm 1 involves several important factors that complicate the estimator design, which cover 1) the time-varying system parameters; 2) the time length of one communication cycle of the FRP; and 3) the bound of non-Gaussian noises.

Remark 7: In this paper, we have made much effort in handling the SMSE problem for a class of time-varying systems over a FlexRay network. Our main results distinguish themselves in the following two aspects: 1) the transmission rules for both static and dynamic scheduling have been established through the mathematical description of the FRP in the discrete-time setting; and 2) the impact from the FRP on the estimation performance has been closely examined in a quantitative way.

\section{An ILlustrative EXAMPle}

In this section, the usefulness of the proposed FlexRaybased state estimator design scheme is validated via a simulation example.

Consider the discrete time-varying system (1) with the following parameters:

$$
\begin{aligned}
& A\left(T_{k}\right)=\left[\begin{array}{cccc}
a_{1}\left(T_{k}\right) & 0.25 & 0 & 0 \\
-0.1 & a_{2}\left(T_{k}\right) & 0 & 0 \\
0.2 & 0 & -0.8 & a_{3}\left(T_{k}\right) \\
0 & 0 & 0.1 & 1
\end{array}\right] \\
& B\left(T_{k}\right)=\left[\begin{array}{c}
0.13+0.2 \sin \left(10 T_{k}\right) \\
0.15-0.2 \cos \left(2 T_{k}\right) \\
0.2 \\
-0.15
\end{array}\right] \\
& C\left(T_{k}\right)=\left[\begin{array}{cccc}
c_{1}\left(T_{k}\right) & 0 & 0 & 0 \\
0 & c_{2}\left(T_{k}\right) & 0 & 0 \\
0 & 0 & 6 & 0 \\
0 & 0 & 0 & 8
\end{array}\right] \\
& D\left(T_{k}\right)=\left[\begin{array}{c}
0.12 \\
0.17+0.27 \cos \left(T_{k}\right) \\
0.2 \\
-0.2
\end{array}\right]
\end{aligned}
$$

where

$$
\begin{aligned}
& a_{1}\left(T_{k}\right)=1.19+0.2 \sin \left(10 T_{k}\right), \\
& a_{2}\left(T_{k}\right)=0.16-0.2 \cos \left(2 T_{k}\right), \\
& a_{3}\left(T_{k}\right)=-0.1 \sin \left(4 T_{k}\right), \\
& c_{1}\left(T_{k}\right)=9+0.3 \cos \left(-2 T_{k}\right), \\
& c_{2}\left(T_{k}\right)=1.8-0.1 \cos \left(3 T_{k}\right) .
\end{aligned}
$$

The initial state and its initial estimation are, respectively taken as $x(0)=\left[\begin{array}{llll}0.15 & 0.16 & 0.1 & -0.2\end{array}\right]^{T}$ and $\left[\begin{array}{llll}0 & 0 & 0 & 0\end{array}\right]^{T}$. The non-Gaussian noise is set as $w\left(T_{k}\right)=0.3 \sin \left(-T_{k}\right)$. Let the initial value of $P\left(t_{k}\right)$ be $P(0)=I$. The weighting matrices are chosen as $W\left(T_{k}\right)=10, Q_{3}\left(T_{k}\right)=2$ and $Q_{4}\left(T_{k}\right)=1$. The relationship of $t_{k}$ and $T_{k}$ satisfies $t_{k+1}-t_{k}=3\left(T_{k+1}-T_{k}\right)$. 
This article has been accepted for publication in a future issue of this journal, but has not been fully edited. Content may change prior to final publication. Citation information: DOI10.1109/TSMC.2021.3071390, IEEE Transactions on Systems, Man, and Cybernetics: Systems

The step-size is $N=39$. The obtained state estimator gains at time $t_{1}, t_{2}$ and $t_{3}$ are listed in Tables I-III, respectively.

The optimization problem is solved by the MATLABR2018B software performed on a 3.60GHz quad-core desktop computer with 16GB RAM using 64bit. Under this experimental setup, the solution of the addressed optimization problem takes nearly $4.0866 \mathrm{~s}$, which mainly causes by the use of the lifting technique and the vector augmentation method. The simulation results are shown in Figs. 2-7. More specifically, the curves of the true states and their estimates are depicted in Figs. 2-5. The curves of the estimation errors and their upper bounds are plotted in Figs. 6-7. It can be concluded that the simulation results show the usefulness of the developed protocol-based estimator design scheme.

TABLE I: The state estimator parameter at time $t_{1}$

\begin{tabular}{|c|c|c|c|c|}
\hline$k$ & \multicolumn{4}{|c|}{$L\left(t_{k}\right)$} \\
\hline \multirow{14}{*}{$k=1$} & 0.1268 & -0.1483 & $\overline{-0.0043}$ & -0.0213 \\
\hline & -0.0105 & 0.0560 & 0.0025 & 0.0088 \\
\hline & 0.0269 & -0.0011 & -0.0581 & -0.0302 \\
\hline & 0.0065 & 0.4176 & 0.0047 & 0.1192 \\
\hline & 0.1358 & -0.0864 & -0.0044 & -0.0220 \\
\hline & 0.0000 & 0.6882 & -0.0024 & -0.0081 \\
\hline & 0.0169 & 0.5686 & 0.0430 & 0.0183 \\
\hline & 0.0000 & -0.0069 & 0.0011 & 0.1241 \\
\hline & 0.1839 & -0.0325 & 0.0070 & -0.0432 \\
\hline & -0.0159 & 0.1314 & 0.0120 & -0.0100 \\
\hline & 0.0122 & -0.5265 & -0.0267 & -0.0383 \\
\hline & 0.0026 & 0.0913 & -0.0011 & 0.1312 \\
\hline & 0.0000 & 0.0000 & 1.0000 & 0.0000 \\
\hline & 0.0000 & 0.0000 & 0.0000 & 1.0000 \\
\hline
\end{tabular}

TABLE II: The state estimator parameter at time $t_{2}$

\begin{tabular}{|c|c|c|c|c|}
\hline$k$ & \multicolumn{4}{|c|}{$L\left(t_{k}\right)$} \\
\hline \multirow{14}{*}{$k=2$} & 0.1539 & 1.509 & $\overline{-0.0022}$ & -0.0379 \\
\hline & 0.0000 & 0.6249 & 0.0000 & -0.0025 \\
\hline & -0.0630 & -2.4464 & -0.0471 & 0.0291 \\
\hline & 0.0213 & 1.9819 & 0.0000 & 0.0174 \\
\hline & 0.2325 & 5.4709 & -0.0109 & -0.2396 \\
\hline & 0.0016 & 1.9455 & -0.0049 & -0.1175 \\
\hline & 0.1007 & 4.5693 & 0.0316 & -0.1652 \\
\hline & 0.0000 & 0.0685 & 0.0000 & 0.1215 \\
\hline & 0.2674 & 6.9222 & -0.0150 & -0.3128 \\
\hline & -0.0123 & 0.9621 & -0.0064 & -0.0593 \\
\hline & -0.0276 & -2.0192 & -0.0311 & 0.0450 \\
\hline & 0.0060 & 0.1142 & 0.0055 & 0.1261 \\
\hline & 0.0000 & 0.0000 & 1.0000 & 0.0000 \\
\hline & 0.0000 & 0.0000 & 0.0000 & 1.0000 \\
\hline
\end{tabular}

\section{CONCLUSIONS}

In this paper, the SMSE issue has been dealt with for a class of discrete-time networked systems under the FRP. By slowing the update rate of the state estimator, a multi-rate model has been put forward to characterize the effect of the FRP on the estimation performance. By virtue of the lifting technique combined with the vector augmentation method, the multi-rate system has been transformed into a single rate one where the update rate is the same with one communication period of the FRP. By means of the convex optimization approach, an optimized ellipsoid has been obtained by solving an optimization problem with certain inequality constraints.

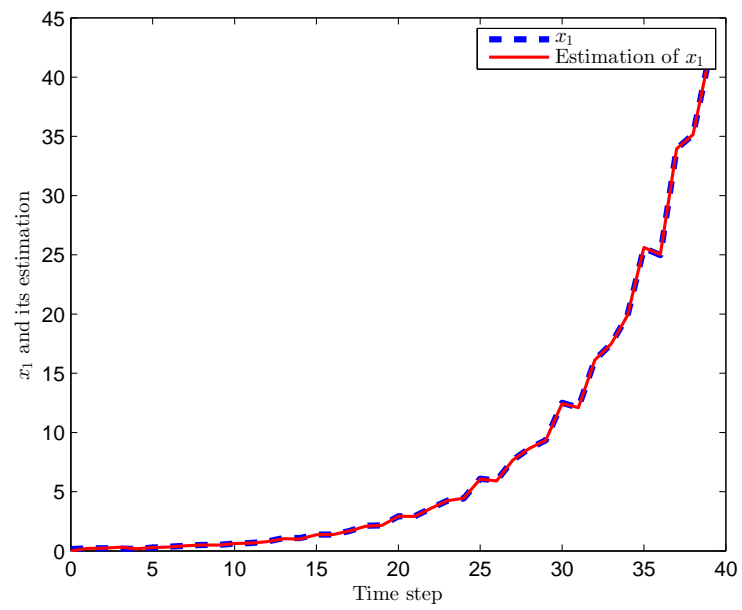

Fig. 2: State $x_{1}$ and its estimation

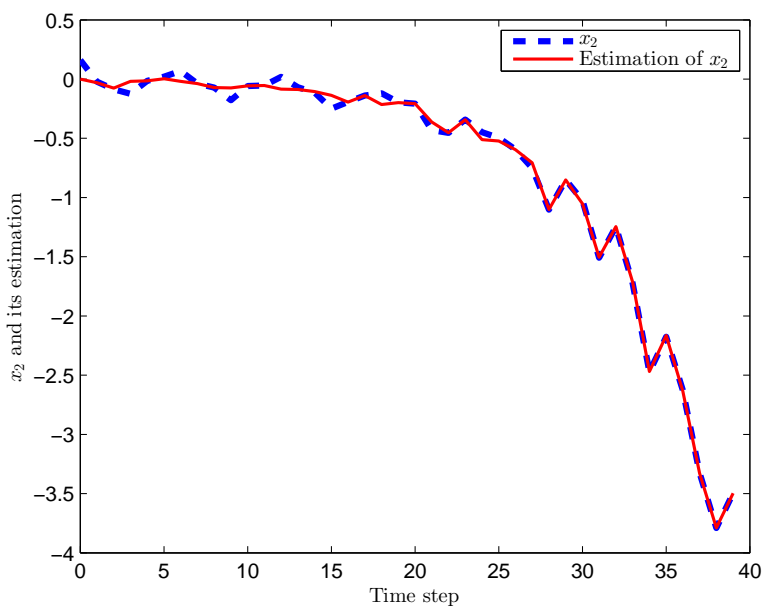

Fig. 3: State $x_{2}$ and its estimation



Fig. 4: State $x_{3}$ and its estimation 
This article has been accepted for publication in a future issue of this journal, but has not been fully edited. Content may change prior to final publication. Citation information: DOI10.1109/TSMC.2021.3071390, IEEE Transactions on Systems, Man, and Cybernetics: Systems

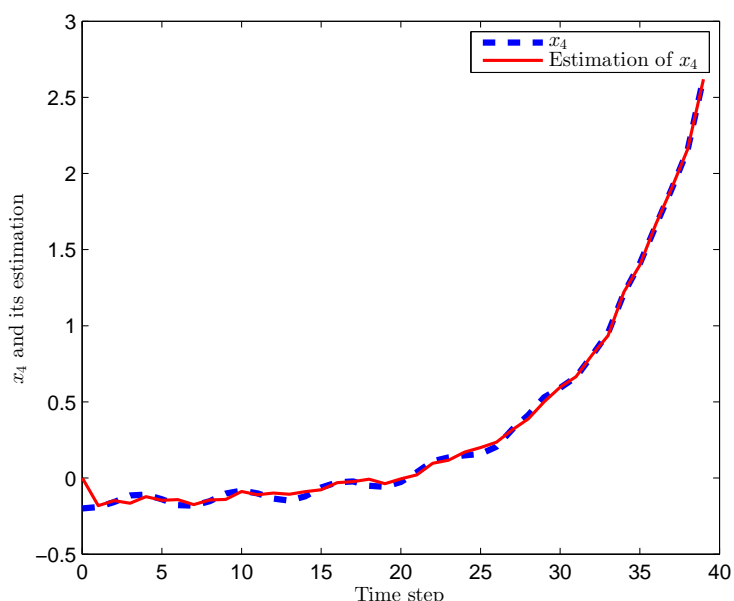

Fig. 5: State $x_{4}$ and its estimation


Fig. 6: The estimate errors $e_{1}$ and $e_{2}$ as well as their upper bounds
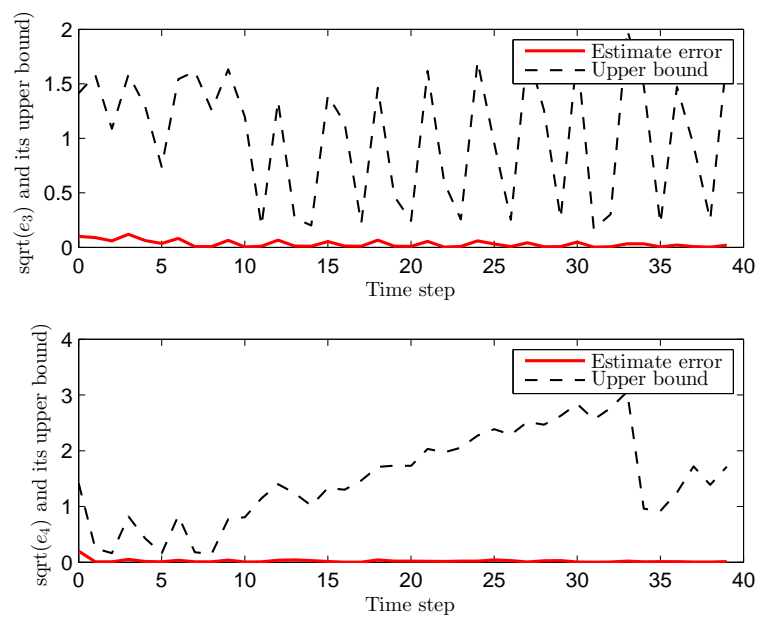

Fig. 7: The estimate errors $e_{3}$ and $e_{4}$ as well as their upper bounds
TABLE III: The state estimator parameter at time $t_{3}$

\begin{tabular}{|c|c|c|c|c|}
\hline$k$ & \multicolumn{4}{|c|}{$L\left(t_{k}\right)$} \\
\hline \multirow{14}{*}{$k=3$} & $\overline{0.1211}$ & $\overline{-0.2321}$ & $\overline{0.0061}$ & -0.0670 \\
\hline & -0.0116 & 0.0122 & 0.0038 & 0.0073 \\
\hline & 0.0174 & 0.4863 & -0.1212 & 0.0383 \\
\hline & 0.0067 & 0.3423 & 0.0095 & 0.1154 \\
\hline & 0.1764 & 0.4361 & 0.0038 & -0.0986 \\
\hline & -0.0066 & 0.3458 & -0.0024 & 0.0032 \\
\hline & 0.0217 & 0.0786 & 0.0941 & -0.0544 \\
\hline & 0.0000 & -0.0016 & 0.0000 & 0.1246 \\
\hline & 0.1737 & 0.5230 & 0.0027 & -0.0959 \\
\hline & -0.0225 & 0.0565 & 0.0012 & 0.0058 \\
\hline & 0.0164 & 0.0119 & -0.0731 & 0.0139 \\
\hline & 0.0031 & 0.0157 & 0.0085 & 0.1215 \\
\hline & 0.0000 & 0.0000 & 1.0000 & 0.0000 \\
\hline & 0.0000 & 0.0000 & 0.0000 & 1.0000 \\
\hline
\end{tabular}

An illustrative example has been provided to show the effectiveness of the proposed protocol-based state estimator design scheme. Further research topics include the extension of our results to 1) other FRP-based state estimation problems, such as the $H_{\infty}$ filtering, the Kalman filtering and the extended Kalman filtering [12], [22], [29]; and 2) more general systems, such as complex networks, sensor networks and multi-agent systems [16], [17].

\section{REFERENCES}

[1] M. Basin and M. Hernandez-Gonzalez, Discrete-time $H_{\infty}$ filtering for nonlinear polynomial systems, International Journal of Systems Science, vol. 47, no. 9, pp. 2058-2066, Jul. 2016.

[2] S. Boyd, L. Ghaoui, E. Feron and V. Balakrishnan, Linear matrix inequalities in system and control theory, Philadelphia, PA: SIAM, 1994.

[3] R. Caballero-Aguila, A. Hermoso-Carazo and J. Linares-Perez, Distributed fusion filters from uncertain measured outputs in sensor networks with random packet losses, Information Fusion, vol. 34, pp. 7079, Mar. 2017.

[4] Y. Chen, Z. Wang, L. Wang and W. Sheng, Finite-horizon $H_{\infty}$ state estimation for stochastic coupled networks with random inner couplings using Round-Robin protocol, IEEE Transactions on Cybernetics, in press, DOI: 10.1109/TCYB.2020.3004288.

[5] D. Ciuonzo, A. Aubry, and V. Carotenuto, Rician MIMO channel- and jamming-aware decision fusion, IEEE Transactions on Signal Processing, vol. 65 , no. 15 , pp. 3866-3880, 2017.

[6] P. Cui, H. Zhang, J. Lam and L. Ma, Real-time Kalman filtering based on distributed measurements, International Journal of Robust and Nonlinear Control, vol. 23, no. 14, pp. 1597-1608, Sept. 2013.

[7] D. Ding, Q.-L. Han, Y. Xiang, X. Ge and X.-M. Zhang, A survey on security control and attack detection for industrial cyber-physical systems, Neurocomputing, vol. 275, pp. 1674-1683, Jan. 2018.

[8] D. Ding, Z. Wang and Q.-L. Han, A set-membership approach to eventtriggered filtering for general nonlinear systems over sensor networks, IEEE Transactions on Automatic Control, vol. 65, no. 4, pp. 1792-1799, Apr. 2020

[9] H. Dong, X. Bu, Z. Wang and F. Han, Finite-horizon distributed state estimation under randomly switching topologies and redundant channels, IEEE Transactions on Systems Man Cybernetics-Systems, vol. 50, no. 8, pp. 2938-2947, Aug. 2020.

[10] X. Ge, Q.-L. Han, X.-M. Zhang, L. Ding and F. Yang, Distributed eventtriggered estimation over sensor networks: A survey, IEEE Transactions on Cybernetics, vol. 50, no. 3, pp. 1306-1320, Mar. 2020.

[11] H. Geng, Y. Liang, Y. Liu and F. E. Alsaadi, Bias estimation for asynchronous multi-rate sensor fusion with unknown inputs, Information Fusion, vol. 39, pp. 139-153, Jan. 2018.

[12] H. Geng, Z. Wang, L. Zou, A. Mousavi and Y. Cheng, Protocolbased Tobit Kalman filter under integral measurements and probabilistic sensor failures, IEEE Transactions on Signal Processing, in press, DOI: 10.1109/TSP.2020.3048245.

[13] Z. Gu, P. Shi, D. Yue and Z. Ding, Decentralized adaptive event-triggered $H_{\infty}$ filtering for a class of networked nonlinear interconnected systems, IEEE Transactions on Cybernetics, vol. 49, no. 5, pp. 1570-1579, 2018. 
This article has been accepted for publication in a future issue of this journal, but has not been fully edited. Content may change prior to final publication. Citation information: DOI10.1109/TSMC.2021.3071390, IEEE Transactions on Systems, Man, and Cybernetics: Systems

[14] W. P. M. H. Heemels, A. R. Teel, N. van de Wouw and D. Nešić, Networked control systems with communication constraints: tradeoffs between transmission intervals, delays and performance, IEEE Transactions on Automatic Control, vol. 55, no. 8, pp. 1781-1796, Sept. 2010

[15] N. Hou, H. Dong, Z. Wang and H. Liu, A partial-node-based approach to state estimation for complex networks with sensor saturations under random access protocol, IEEE Transactions on Neural Networks and Learning Systems, DOI:10.1109/TNNLS.2020.3027252.

[16] J. Hu, Z. Wang, G.-P. Liu, H. Zhang and R. Navaratne, A predictionbased approach to distributed filtering with missing measurements and communication delays through sensor networks, IEEE Transactions on Systems, Man, and Cybernetics: Systems, in press, DOI: 10.1109/TSMC.2020.2966977.

[17] J. Hu, Z. Wang, G.-P. Liu and H. Zhang, Variance-constrained recursive state estimation for time-varying complex networks with quantized measurements and uncertain inner coupling, IEEE Transactions on Neural Networks and Learning Systems, vol. 31, no. 6, pp. 1955-1967, 2020.

[18] S. Hu, D. Yue, Q.-L. Han, X. Xie, X. Chen and C. Dou, Observer-based event-triggered control for networked linear systems subject to denialof-service attacks, IEEE Transactions on Cybernetics, vol. 50, no. 5 , pp. 1952-1964, 2019.

[19] O. N. Kiselev and B. T. Polyak, Ellipsoidal estimation with respect to a generalized criterion, Automation and Remote Control, vol. 52, pp. 1281-1292, 1992.

[20] Q. Li, Z. Wang, B. Shen and W. Sheng, Recursive distributed filtering over sensor networks on Gilbert-Elliott channels: A dynamic event-triggered approach, Automatica, DOI: 10.1016/j.automatica.2019.108681.

[21] X. Li, H. Dong, Z. Wang and F. Han, Set-Membership filtering for statesaturated systems with mixed time-delays under weighted Try-OnceDiscard protocol, IEEE Transactions on Circuits and Systems II-Express Briefs, vol. 66, no. 2, pp. 312-316, Feb. 2019.

[22] D. Liu, Z. Wang, Y. Liu and F. E. Alsaadi, Extended Kalman filtering subject to random transmission delays: Dealing with packet disorders, Information Fusion, vol. 60, pp. 80-86, 2020.

[23] J. Liu, M. Yang, E. Tian, J. Cao and S. Fei, Event-based security controller design for state-dependent uncertain systems under hybridattacks and its application to electronic circuits, IEEE Transactions on Circuits and Systems I: Regular Papers, vol. 66, no. 12, pp. 4817-4828, Dec. 2019.

[24] J. Liu, Y. Gu, L. Zha, Y. Liu and J. Cao, Event-triggered $H_{\infty}$ load frequency control for multiarea power systems under hybrid cyber attacks, IEEE Transactions on Systems Man Cybernetics: Systems, vol. 49, no. 8, pp. 1665-1678, Aug. 2019.

[25] S. Liu, Z. Wang, G. Wei and M. Li, Distributed set-membership filtering for multirate systems under the Round-Robin scheduling over sensor networks, IEEE Transactions on Cybernetics, vol. 50, no. 5, pp. 19101920, May 2020.

[26] S. Liu, Z. Wang, Y. Chen and G. Wei, Protocol-based unscented Kalman filtering in the presence of stochastic uncertainties, IEEE Transactions on Automatic Control, vol. 65, no. 3, pp. 1303-1309, 2020.

[27] S. Liu, Z. Wang, L. Wang and G. Wei, $H_{\infty}$ pinning control of complex dynamical networks under dynamic quantization effects: A coupled backward Riccati equation approach, IEEE Transactions on Cybernetics, in press, DOI: 10.1109/TCYB.2020.3021982.

[28] Y. Liu, Z. Wang, L. Ma and F. E. Alsaadi, A partial-nodes-based information fusion approach to state estimation for discrete-time delayed stochastic complex networks, Information Fusion, vol. 49, pp. 240-248, Sept. 2019.

[29] Y. Liu, B. Shen and H. Shu, Finite-time resilient $H_{\infty}$ state estimation for discrete-time delayed neural networks under dynamic event-triggered mechanism, Neural Networks, vol. 121, pp. 356-365, Jan. 2020.

[30] L. Ma, Z. Wang, Y. Liu and F. E. Alsaadi, Distributed filtering for nonlinear time-delay systems over sensor networks subject to multiplicative link noises and switching topology, International Journal of Robust and Nonlinear Control, vol. 29, no. 10, pp. 2941-2959, 2019.

[31] R. Makowitz and C. Temple, FlexRay-a communication network for automotive control systems, in 2006 IEEE International Workshop on Factory Communication Systems, June 2006, pp. 207-212.

[32] D. G. Meyer, A new class of shift-varying operators, their shiftinvariant equivalents, and multirate digital systems, IEEE Transactions on Automatic Control, vol. 35, no. 4, pp. 429-433, Apr. 1990.

[33] W. Qian, Y. Li, Y. Chen, and W. Liu, $L_{2}-L_{\infty}$ filtering for stochastic delayed systems with randomly occurring nonlinearities and sensor saturation, International Journal of Systems Science, vol. 51, no. 13, pp. 2360-2377, 2020
[34] F. C. Schweppe, Recursive state estimation: Unknown but bounded errors and system inputs, IEEE Transactions on Automatic Control, vol. AC-13, no. 1, pp. 102-107, 1968.

[35] B. Shen, Z. Wang, D. Wang, J. Luo, H. Pu and Y. Peng, Finite-horizon filtering for a class of nonlinear time-delayed systems with an energy harvesting sensor, Automatica, vol. 100, pp. 144-152, Feb. 2019.

[36] Y. Shen, Z. Wang, B. Shen and H. Dong, Outlier-resistant recursive filtering for multi-sensor multi-rate networked systems under weighted Try-Once-Discard protocol, IEEE Transactions on Cybernetics, in press, DOI: $10.1109 /$ TCYB.2020.3021194.

[37] Y. Shen, Z. Wang, B. Shen and Q.-L. Han, Recursive state estimation for networked multi-rate multi-sensor systems with distributed time-delays under the Round-Robin protocol, IEEE Transactions on Cybernetics, in press, DOI: 10.1109/TCYB.2020.3021350.

[38] H. Tan, B. Shen, Y. Liu, A. Alsaedi and B. Ahmad, Event-triggered multi-rate fusion estimation for uncertain system with stochastic nonlinearities and colored measurement noises, Information Fusion, vol. 36, pp. 313-320, 2017

[39] Y. Tang, D. Zhang, D. W. C. Ho and F. Qian, Tracking control of a class of cyber-physical systems via a FlexRay communication network, IEEE Transactions on Cybernetics, vol. 49, no. 4, pp. 1186-1199, Apr. 2019.

[40] F. Wang and J. Liang, Constrained $H_{\infty}$ estimation for time-varying networks with hybrid incomplete information, International Journal of Robust and Nonlinear Control, vol. 28, no. 2, pp. 699-715, 2018.

[41] L. Wang, Z. Wang, B. Shen and G. Wei, Recursive filtering with measurement fading: A multiple description coding scheme, IEEE Transactions on Automatic Control, DOI: 10.1109/TAC.2020.3034196.

[42] L. Wang, Z. Wang, G. Wei and F. E. Alsaadi, Observer-based consensus control for discrete-time multi-agent systems with coding-decoding communication protocol, IEEE Transactions on Cybernetics, vol. 49, no. 12 , pp. $4335-4345,2019$

[43] W. Wang, D. Nešić and R. Postoyan, Emulation-based stabilization of networked control systems implemented on FlexRay, Automatica vol. 59, pp. 73-83, Sept. 2015.

[44] W. Wang, D. Nešić and R. Postoyan, Observer design for networked control systems with FlexRay, Automatica, vol. 82, pp. 42-48, Aug. 2017.

[45] G. Wei, S. Liu, Y. Song and Y. Liu, Probability-guaranteed setmembership filtering for systems with incomplete measurements, $A u$ tomatica, vol. 60, pp. 12-16, 2015.

[46] Z. Zhao, Z. Wang, L. Zou and J. Guo, Set-Membership filtering for timevarying complex networks with uniform quantisations over randomly delayed redundant channels, International Journal of Systems Science, vol. 51, no. 16, pp. 3364-3377, 2020.

[47] L. Zou, Z. Wang, Q.-L. Han and D. H. Zhou, Moving horizon estimation of networked nonlinear systems with random access protocol, IEEE Transactions on Systems, Man, and Cybernetics: Systems, in press, DOI: 10.1109/TSMC.2019.2918002.

[48] L. Zou, Z. Wang, H. Geng and X. Liu, Set-membership filtering subject to impulsive measurement outliers: A recursive algorithm, IEEE/CAA Journal of Automatica Sinica, vol. 8, no. 2, pp. 377-388, 2021.

[49] L. Zou, Z. Wang, H. Dong and Q.-L. Han, Moving horizon estimation with multirate measurements and correlated noises, International Journal of Robust and Nonlinear Control, vol. 30, no. 17, pp. 7429-7445, 2020 .

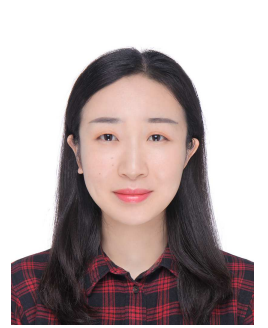

Shuai Liu received the Ph.D. degree in system analysis and integration from the University of Shanghai for Science and Technology, Shanghai, China, in 2018

From 2017 to 2018, she was a visiting Ph.D. student with the Department of Computer Science, Brunel University London, Uxbridge, U.K. She is currently a Post-Doctoral Research Fellow with the College of Science, University of Shanghai for Science and Technology, Shanghai, China. She is a recipient of National Postdoctoral Innovative Talent Scholars of China. She has published over ten papers in refereed international journals. Her current research interests include sensor networks, setmembership filtering, model predictive control as well as Kalman filtering.

Dr. Liu is currently a reviewer for some international journals. 


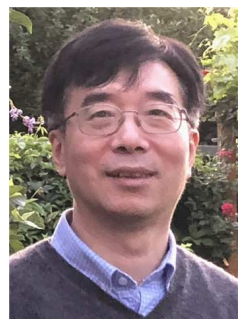

Zidong Wang (SM'03-F'14) was born in Jiangsu, China, in 1966. He received the B.Sc. degree in mathematics in 1986 from Suzhou University, Suzhou, China, and the M.Sc. degree in applied mathematics in 1990 and the Ph.D. degree in electrical engineering in 1994, both from Nanjing University of Science and Technology, Nanjing, China.

$\mathrm{He}$ is currently a Professor of Dynamical Systems and Computing in the Department of Computer Science, Brunel University London, U.K. From 1990 to 2002, he held teaching and research appointments in universities in China, Germany and the UK. Prof. Wang's research interests include dynamical systems, signal processing, bioinformatics, control theory and applications. He has published around 600 papers in refereed international journals. He is a holder of the Alexander von Humboldt Research Fellowship of Germany, the JSPS Research Fellowship of Japan, William Mong Visiting Research Fellowship of Hong Kong.

Prof. Wang serves (or has served) as the Editor-in-Chief for International Journal of Systems Science, the Editor-in-Chief for Neurocomputing, and an Associate Editor for 12 international journals including IEEE Transactions on Automatic Control, IEEE Transactions on Control Systems Technology, IEEE Transactions on Neural Networks, IEEE Transactions on Signal Processing, and IEEE Transactions on Systems, Man, and Cybernetics-Part C. He is a Member of the Academia Europaea, a Fellow of the IEEE, a Fellow of the Royal Statistical Society and a member of program committee for many international conferences.

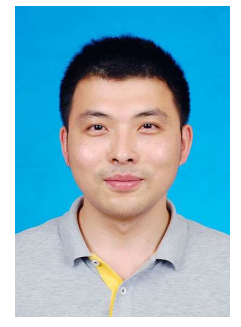

Licheng Wang received the B.Sc. degree in automation in 2011 from Weifang University, Weifang, China, and the M.Sc. degree and the Ph.D. degree in control science and engineering from University of Shanghai for Science and Technology, Shanghai, China, in 2014 and 2019, respectively.

Since Nov. 2016-Nov. 2018, he has been a visiting $\mathrm{Ph} . \mathrm{D}$. student in the Department of Electronic and Computer Engineering at Brunel University London in the UK. He is currently a Post-Doctoral Research Fellow with the Department of Control Science and Engineering, University of Shanghai for Science and Technology, Shanghai, China. His research interests include nonlinear stochastic control and filtering, as well as complex networks and sensor networks.

Mr. Wang is currently a reviewer for some international journals.

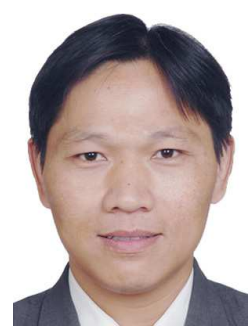

Guoliang Wei received the B.Sc. degree in mathematics from Henan Normal University, Xinxiang, China, in 1997 and the M.Sc. degree in applied mathematics and the Ph.D. degree in control engineering, both from Donghua University, Shanghai, China, in 2005 and 2008, respectively. He is currently a Professor with the Department of Control Science and Engineering, University of Shanghai for Science and Technology, Shanghai, China.

From March 2010 to May 2011, he was an Alexander von Humboldt Research Fellow in the Institute for Automatic Control and Complex Systems, University of DuisburgEssen, Germany. From March 2009 to February 2010, he was a post doctoral research fellow in the Department of Information Systems and Computing, Brunel University, Uxbridge, U.K., sponsored by the Leverhulme Trust of the U.K.. From June to August 2007, he was a Research Assistant at the University of Hong Kong. From March to May 2008, he was a Research Assistant at the City University of Hong Kong.

His research interests include nonlinear systems, stochastic systems, and bioinformatics. He has published more than 100 papers in refereed international journals. He is a very active reviewer for many international journals. 